\title{
A new allele of the Brachytic2 gene in maize can efficiently modify plant architecture
}

\author{
Lai Wei $^{1} \cdot$ Xuan Zhang ${ }^{1} \cdot$ Zhihai Zhang $^{1} \cdot$ Huanhuan Liu' ${ }^{1}$ Zhongwei Lin $^{1}$
}

Received: 10 July 2017 / Revised: 14 November 2017 / Accepted: 16 November 2017 / Published online: 23 February 2018

(c) The Genetics Society 2018

\begin{abstract}
The applications of semi-dwarf genes such as $s d l$ and Rhtl in rice and wheat resulted in the first "green revolution" in the 1960s. However, such semi-dwarf genes that can efficiently reduce plant stature and have few negative yield traits have not yet been identified in maize. In this study, a new allele of Brachytic2 gene (qpal) encoding P-glycoprotein was rapidly finemapped using a modified method. The qpal, containing a 241-bp deletion in the last exon, had no negative effect on yield, but greatly modified the plant architecture including significantly reduced plant height and ear height, increased stalk diameter and erected leaf. A common variant similar to maize qpal was also present in the sorghum orthologous $d w 3$ locus. Comparative RNA-seq analysis next showed 99 differentially co-expressed genes affected by $\mathrm{Br} 2$ in maize and $d w 3$ in sorghum, including four plant height genes D3, BAK1, Actin7 and Csld1, which are involved in gibberellin and brassinosteroid biosynthesis, auxin transport and cellulose synthesis. The qpal can be applied to efficiently modify plant stature in maize and in combination with D3, BAKl, Actin7, Csldl and the other 95 differentially co-expressed genes, can be edited using new genomic editing tools for further applications and studies.
\end{abstract}

\section{Introduction}

As the backbone of plant architecture, the plant stalk builds a bridge between the carbon "source" and "sink" and by determining the height of a plant, can greatly affect cereal yield. During cereal domestication and improvement, plant

These authors contributed equally: Lai Wei, Xuan Zhang

Data archiving The raw RNA-seq data for this study have been submitted to the NCBI Sequence Read Archive (SRA) under accession no. SRP076433. The authors state that all data necessary for confirming the conclusions presented in the article are presented fully within the article.

Accession numbers Sequence Data was deposited in Genbank with the accession numbers MG256511 and MG256512.

Electronic supplementary material The online version of this article (https://doi.org/10.1038/s41437-018-0056-3) contains supplementary material, which is available to authorized users.

Zhongwei Lin

zlin@cau.edu.cn

1 National Maize Improvement Center; Department of Plant Genetics and Breeding, China Agricultural University, Beijing 100193, China height has been intensively reshaped. Cereal landraces are generally very tall and large in stature (Bensen et al. 1995). However, such tall plants and large stature often lead to low planting density and lodging, which both can cause large losses of yield. To prevent lodging in cereal, the selection of cereal with moderate short stature has been pursued in most modern breeding programs. The increases in maize yield per unit area have been primarily due to increased planting density, originated from the application of varieties with moderately short stature (Duvick 2005), during recent decades. Rice and wheat production sharply increased in the 1960 s as the "Green Revolution" popularized the use of semi-dwarf cultivars (Hedden 2003). A reduction in plant height has the potential to increase dry matter accumulation and yield in cereal crops.

Rice and wheat green revolution genes, $s d l$ (Sasaki et al. 2002) involved in the biosynthesis of gibberellin (GA) and Rhtl (Peng et al. 1999) serving as a repressor in the GA signal pathway, effectively shape the tall plant height into semi-dwarf stature. However, because plant height is generally controlled by many quantitative trait loci (QTLs) with tiny genetic effects (Peiffer et al. 2014), the selection efficiency of moderate short varieties remains low in maize. Moreover, several cloned maize dwarf genes that can greatly decrease both plant height and ear size are difficult 
to apply. These maize dwarf genes include Dwarf3 (D3) encoding a cytochrome P450 involved in the early stages of GA biosynthesis (Winkler and Helentjaris 1995), Anther earl (Anl) encoding an enzyme involved in the synthesis of ent-kaurene, the first tetracyclic intermediate in GA biosynthesis (Bensen et al. 1995), and Br2 encoding Pglycoprotein regulating polar auxin transport in the maize stalk (Multani et al. 2003). Although maize possesses a tremendous genetic diversity, the favorable alleles of plant height genes, which can effectively change the plant height and have no significant negative effect on yield traits similar to $s d l$ and $R h t l$, have not yet been identified in maize.

In this study, we first identified a major QTL of plant height on maize chromosome 1 (qpal) in an $\mathrm{F}_{2}$ population with a high-density genetic map of single-nucleotide polymorphisms (SNPs) from GBS (genotyping by sequencing (GBS)). This major QTL was then rapidly fine-mapped within a segment of $354 \mathrm{~kb}$ using a marker test performed in the $\mathrm{F}_{3: 4}$ population. Comparative genomic and sequencing analysis further revealed that a new allele of the $B r 2$ gene (qpal) with a 241-bp deletion was present in the fifth exon, leading to the loss of the second P-loop NTPase domain. Large phenotype analysis then showed that qpal could greatly reshape the plant architecture with few negative yield traits. Similar change to maize qpal was also present in the sorghum $d w 3$ locus. Comparative RNA-seq analysis next showed 99 differentially co-expressed genes affected by $b r 2$ in maize and $d w 3$ in sorghum, including four plant height genes D3, BAKl, Actin7 and Csdll, which are involved in GA and brassinosteroid (BR) biosynthesis, auxin transport and cellulose synthesis. The $B r 2$ gene can work with D3, BAKl, Actin 7 and Csld1 and regulate multiple plant hormones to govern plant height. The rapid finemapping strategy of a QTL can greatly accelerate the cloning of major QTLs, such as qpal. Unlike several reported alleles of the $B r 2$ gene, our newly identified qpal can be efficiently applied to modify crop plant architecture, and the 99 co-expressed genes can be used to further reshape plant height and explore the molecular basis of plant height.

\section{Materials and methods}

\section{Plant materials}

The short and tall maize inbred lines 52220 and Mo18W, respectively, were crossed to construct $\mathrm{F}_{2}$ and $\mathrm{F}_{3: 4}$ populations. 52220 is a breeding line developed by Professor Tongming Song, National Maize Improvement Center, China Agricultural University. Sorghum virgatum (SV; synonym S. bicolor subsp. verticilliflorum race virgatum) is native to Egypt and exhibits typical wild grass characteristics such as shattering, multiple branching and early flowering. SV was crossed with a domesticated sorghum (Sorghum bicolor (L.) Moench) commercial line Tx623 to construct $\mathrm{F}_{2}, \mathrm{~F}_{3}$ and $\mathrm{F}_{4}$ recombinant inbred line (RIL) populations. Twelve plants of each maize and sorghum $\mathrm{F}_{2}$, $\mathrm{F}_{3}$ and $\mathrm{F}_{4} \mathrm{RIL}$ were grown in $12 \times 50$ (row by row) $\times 25$ (plant by plant) $\mathrm{cm}$ plots at experimental stations of China Agricultural University in Beijing and Hainan between 2013 and 2016. The phenotypic data for ten traits in the global maize natural population with 527 inbred lines were downloaded from http://maizego.org/Resources.html (Yang et al. 2014; Yang et al. 2011).

\section{GBS analysis}

GBS analysis was performed following the method described by Elshire et al. (2011) with some modifications. Genomic DNA was extracted from leaves of the $F_{2}$ population with 281 individuals and the two parents using a modified CTAB method. The purified genomic DNA was first digested by ApeKI (New England Biolabs, Ipswitch, MA) to reduce the complexity of the maize genome. Ligated with 4-8 bases of bar-code adapters for distinction, the digested DNA was then combined and purified with a QIAquick PCR Purification Kit (Qiagen). Enrichment of libraries containing 170-350-bp maize digested DNA was subsequently conducted through PCR with a Phusion ${ }^{\circ}$ High-Fidelity PCR Kit (New England Biolabs, Ipswitch, MA) and a pair of primers: 5'-AATGATACGGCGACCACCGAGATCTACACTCTTTCC-

CTACACGACGCTCTTCCGATCT-3' and 5'-CAAGCAGAAGACGGCATACGAG-ATCGGTCTCGGCATTCCTGCTGAACCGCTCTTCCGATCT- ${ }^{\prime}$. The libraries were finally sequenced using an Illumina Hiseq2000 sequencer. The two parent lines 52220 and Mo18W were sequenced for approximately $1 \times$ coverage of the whole maize genome, whereas $\mathrm{F}_{2}$ lines were sequenced for an average of $0.05 \times$ coverage. The total of $30 \mathrm{~Gb}$ of raw reads was sorted based on bar-code adapters and then aligned with the B73 whole genome sequence (RefGen_v3, http://www.maizegdb.org) to identify SNPs with BWA (Langmead et al. 2009; Li and Durbin 2009) and a Genome Analysis Toolkit (McKenna et al. 2010).

Although GBS can rapidly detect thousands of SNPs, genetic map construction in the $\mathrm{F}_{2}$ population genotyped using the GBS method needs to resolve three problems: a few SNPs due to sequence errors, a relatively large proportion of missing data in the whole population and a relatively high genotype error rate at heterozygous sites due to low sequencing depth. Most of erroneous or missing genotypes can be corrected with a sliding-window approach 
with a window size of 25 SNPs and a step size of two SNPs (Huang et al. 2009). The ratio of SNP alleles from the two parent lines 52220 and Mo18W in each sliding window was used to determine the genotype of each individual. Those individuals containing the ratio of SNP alleles from either 52220 or Mo18W in each window that reached 18/25 were scored as homozygous 52220 or Mo18W genotypes, whereas the individuals with the ratio below 18/25 were identified as heterozygous genotypes. Adjacent windows with same genotypes were combined into blocks, and the recombinant break points were assumed to be at the boundary of adjacent blocks with different genotypes. Any blocks less than $5 \mathrm{Mb}$ were removed. Next, the genotypic maps of individual $\mathrm{F}_{2} \mathrm{~S}$ over $100-\mathrm{kb}$ intervals were aligned and compared, and a bin-map was generated. Consecutive $100-\mathrm{kb}$ intervals that lacked a recombination event within the population were joined into bins. These bins were then used as markers to construct a high-density genetic map using the function est.map from the R/qtl package.

\section{QTL mapping}

The phenotypic data and genetic map were analyzed with $R /$ qtl using a multiple-QTL mapping method (Broman et al. 2003). Initial, simple interval mapping was performed with the R/qtl function scanone using the Haley-Knott regression method, and a significance threshold at $P=0.05$ for each trait was determined with 1000 permutations. Next, refining the positions of QTLs with logarithm of odds (LOD) score above threshold was performed with the R/qtl function refineqtl. Subsequently, additional QTLs were scanned with the function addqtl based on the refined QTLs. When significant additional QTLs with LOD score above the threshold were detected, the new QTLs would be added into the model, and all the positions of QTLs would be refined again. The cycle of above steps would be repeated until no significant QTLs were added. When all positions of QTLs were refined, the genetic effect and the significance of each QTL were detected with drop-one-QTL analysis in the full model.

\section{QTL fine mapping}

To fine-map the major QTLs of plant height on maize chromosome 1 (qpal), two SSR markers P1 and P7 were used to screen an $\mathrm{F}_{3}$ population for recombination plants with 5215 plants. Then, 697 recombination plants were genotyped with 9 new SSR markers, and 37 representative recombinant plants that harbored homozygous/heterozygous segments at this locus according to these $11 \mathrm{SSR}$ markers were subsequently self-crossed to construct $F_{4}$ families for marker tests between phenotypes and genotypes. In each $\mathrm{F}_{4}$ family, mostly over 100 plants, the marker test was performed with a simple linear regression model to identify the presence or absence of qpal within the heterozygous chromosomal fragment. A significant F-statistic value signified that qpal was located within the heterozygous fragment. Otherwise, qpal was placed in the homozygous fragment. The marker tests were conducted with the $\mathrm{R}$ program, and qpal was specified to a $354-\mathrm{kb}$ segment on chromosome 1, flanked by V2 and V4. The primers of the markers used for genotyping are listed in supplementary table s6.

\section{Comparative genomic analysis}

SYNMAP from the CoGe database (http://genomevolution. org/CoGe/) (Freeling 2009) was used to perform pairwise genomic sequence comparisons. The syntenic map was plotted using the $\mathrm{R}$ program (http://www.r-project.org) based on the BLAST results of pairwise genomes derived from the following data sets in CoGe: sorghum (Tx623, id331), maize (B73, id333), rice (Nipponbare, id3), and foxtail millet (Yugu1, id32546).

\section{Large phenotype comparisons between the $\mathrm{Br} 2$ and qpa1 RIL in maize}

The $\mathrm{F}_{4}$ plants originating from self-pollination of a single plant from the $\mathrm{F}_{3}$ line with heterozygous genomic fragment at qpal locus and harboring either homozygous $\mathrm{Br} 2$ or homozygous qpal alleles, were differentiated using specific primers of qpal. Because most genomic regions were homozygous in the $\mathrm{F}_{4}$ generation, plants homozygous for either $B r 2$ or qpal alleles could be used as near isogenic lines. The loci in the heterozygous regions $(12.5 \%)$ were in balnce in the $\mathrm{F}_{4}$ plants with either $B r 2$ or qpal alleles. The mean value for each trait in the $\mathrm{F}_{4}$ plants with either $B r 2$ or qpal alleles were calculated, and then the effect for each locus in these heterozygous regions was counteracted. Thus, the mean value of each trait represented the effect from either $B r 2$ or qpal alleles. Student's $t$-tests $(n>25)$ were used to compare 13 traits between these two types of RILs with either $\mathrm{Br} 2$ or qpal alleles under similar genetic background, including plant height, ear height, stalk diameter, leaf length, leaf angle, leaf number above ear, tassel primary branch number, tassel length, ear shank length, ear diameter, ear length, kernel row number and husk leaf number.

\section{Comparative RNA-seq analysis}

RNA samples with two replications were collected from the stalks of maize and sorghum RIL plants with $B r 2$ and $b r 2$ at the early (42 days after germination) and middle (56 days after germination) stages of jointing. The total of eight 


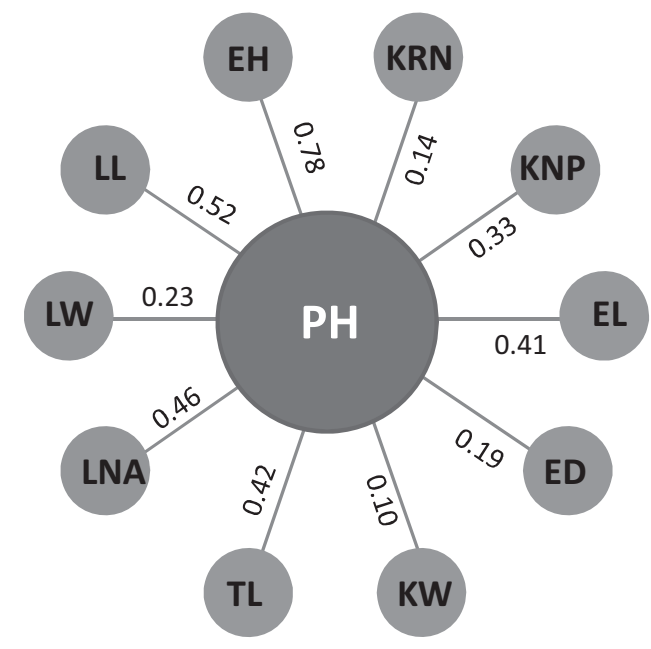

Fig. 1 The correlation network between plant height and other agronomic traits. The agronomic traits include ear height (EH), leaf length (LL), leaf width (LW), leaf number above ear (LNA), tassel length (TL), ear length (EL), ear diameter (ED), kernel row number (KRN), kernel number per row (KNP), and kernel weight (KW). The correlation coefficients are shown on the lines, and the $P$-values of the correlation coefficients for all traits except kernel weight were $<0.05$ (Color figure online)

DNA-free RNA samples was then sequenced with a Hiseq2500 , and $32 \mathrm{~Gb}$ of raw sequencing data were obtained. The raw RNA-seq reads were initially processed to remove the adapter sequences and low-quality bases with Trimmomatic version 0.33 (Bolger et al. 2014) in paired-end mode with recommended parameters. The virus-like and rRNA-like RNA-seq reads were further removed with fastq_clean (Zhang et al., 2014). Finally, the clean RNA-seq reads were mapped to the reference genomes using STAR (Dobin et al. 2013) version 2.5.0b. To improve spliced alignment, STAR was provided with exon junction coordinates from the reference annotations. Default alignment parameters were used, and the outSAMattrIHstart was changed to 0 for compatibility with downstream software Cufflinks. Strong correlations $(r>0.95)$ of gene expression were detected in the biological replicated samples. Gene expression was measured using Cufflinks and cuffdiff 2 (Trapnell et al. 2013), then visualized with the Integrated Genome Browser (Nicol et al. 2009), and fragments per kilo-base of exon per million fragments mapped (FPKM) was obtained. Genes with low expression values $(\mathrm{FPKM}<1)$ were filtered for downstream analysis. The Zea mays reference genome "V3.29" and Sorghum bicolor reference genome "V1.29" with corresponding annotation information were downloaded from Ensenbl Plant (Kersey et al. 2016). The one-toone ortholog genes list between maize and sorghum was obtained from Ensembl BioMarts (Kinsella et al., 2011). Differentially expressed (DE) genes between each compared group were determined by a corrected $P$-value.

\section{Real-time reverse-transcript polymerase chain reaction (RT-PCR) analysis}

Total RNA from each sample was extracted using a plant RNAprep kit (Tiangen Biotech Co. Ltd.), and then the firststrand cDNA was synthesized with an M-MLV first strand kit (Invitrogen). Real-time PCRs were performed for five genes under the inner control of tubulin with three technical replicates, and the transcript level was evaluated with the relative quantification method (Livak and Schmittgen 2001).

\section{Results}

\section{Correlation analysis between plant height and other traits in maize}

To evaluate the effect of plant height on plant architecture and yield component traits in maize, we analyzed the correlations between plant height and ten other traits in a global maize natural population with 527 inbred lines (Fig. 1) (Yang et al. 2014; Yang et al. 2011). Plant height was positively correlated with all ten of these traits, which included ear height, leaf width, leaf angle, leaf length, leaf number above ear, tassel length, ear length, ear diameter, kernel row number and kernel number per row. Plant height was generally more highly correlated with the traits of plant architecture than with the traits of yield components. Plant height strongly influenced ear height $(r=0.78)$ but apparently affected leaf number above ear, leaf length, tassel length and ear length $(0.4<r<0.6)$. However, plant height had almost no effect on leaf width, ear diameter, kernel row number and kernel weight $(r<0.25)$. These results showed that a decrease in plant height was associated with greatly reduced ear height and apparently decreased ear length in maize, which is consistent with mass selection of ear length during maize improvement (Salazar 1985).

\section{Identification of a major QTL of plant height (Br2) using a modified fine-mapping method in maize}

To screen favorable alleles of maize plant height, an extremely short maize breeding line $(52220,73 \mathrm{~cm})$ from the maize genetic germplasm of our center was crossed with an elite maize inbred line Mo18W with tall plant height $(181 \mathrm{~cm})$ to construct an $\mathrm{F}_{2}$ population with 281 lines (Fig. 2a,b). These 281 lines were then genotyped using GBS. A high-density genetic map with 1242 SNPs across the maize ten chromosomes was constructed. The average density in this genetic map reached $1 \mathrm{SNP} / 0.69$ centi-Morgan $(\mathrm{cM})$ (Fig. 2c). 
Fig. 2 Rapid QTL mapping strategy of plant height in maize. a The $F_{2}$ population was constructed with two inbred lines 52220 (short) and Mo18W (tall). Scale bar, $20 \mathrm{~cm}$. b Rapid QTL mapping strategy of plant height: initial QTL mapping was performed in the $\mathrm{F}_{2: 3}$ population with a high-density genetic map of SNPs from GBS, and then QTL fine-mapping was conducted in the $\mathrm{F}_{3: 4}$ population. c High-density genetic map constructed from the $\mathrm{F}_{2}$ population with 281 individuals using GBS (Color figure online) a

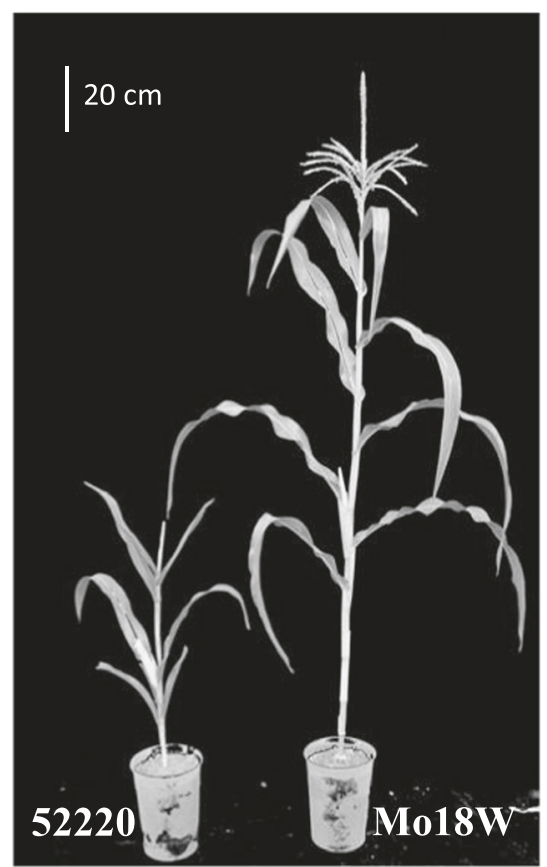

b

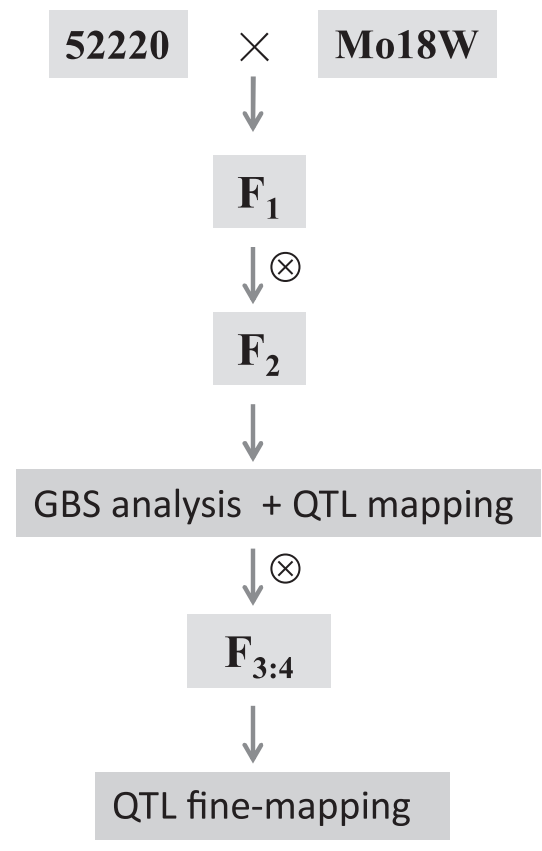

Genetic map

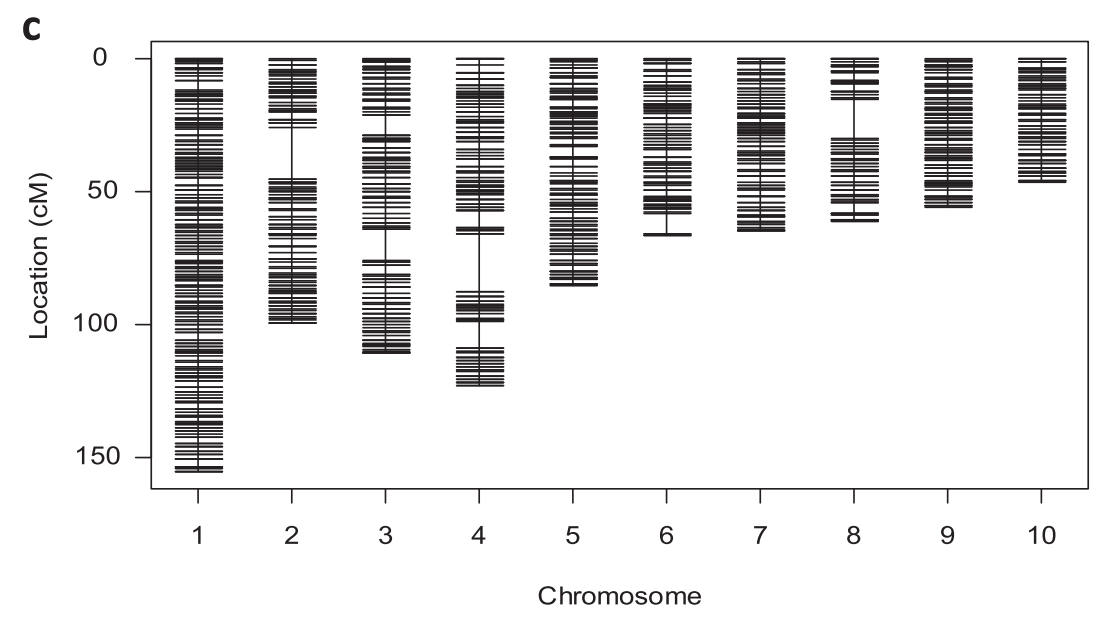

Plant height was approximately normally distributed in the $F_{2}$ population, and QTL mapping in the $F_{2}$ population revealed one major QTL for plant height on maize chromosome 1 in the location from 80 to $110 \mathrm{cM}$, which accounted for $26 \%$ of the total phenotypic variation (Fig. 3a,b), which was successfully confirmed with two SSR markers P1 and P7 on chromosome 1. To fine-map this major QTL of plant height, P1, P7 and 9 new SSRs were used to screen an $\mathrm{F}_{3}$ population with 5215 plants. Thirtyseven representative recombinant plants that harbored homozygous/heterozygous segments at this locus according these 11 SSR markers were subsequently self-crossed to construct $\mathrm{F}_{4}$ families for marker tests between phenotypes and genotypes. With a simple linear regression model, marker tests were then conducted to test the correlations between genotypes and phenotypes in $\mathrm{F}_{4}$ families. Thus, the target gene position in the corresponding $\mathrm{F}_{3}$ recombinants plants was determined (Fig. 3c), which enabled the process of fine mapping. The marker V3 within the heterozygous fragment was strongly correlated with plant height $(P=$ 2.3E-12) in the descendant $\mathrm{F}_{4}$ population with 157 plants derived from the $F_{3}$ plant 17-11, which signified that the target gene was placed on the left side of V4. Similarly, V1 exhibited no association with plant height $(P=0.1590)$ in the descendant $F_{4}$ population from the $F_{3}$ plant 145-9, indicating that the target gene was located on the right side of V2. Therefore, this major QTL of plant height was finally 

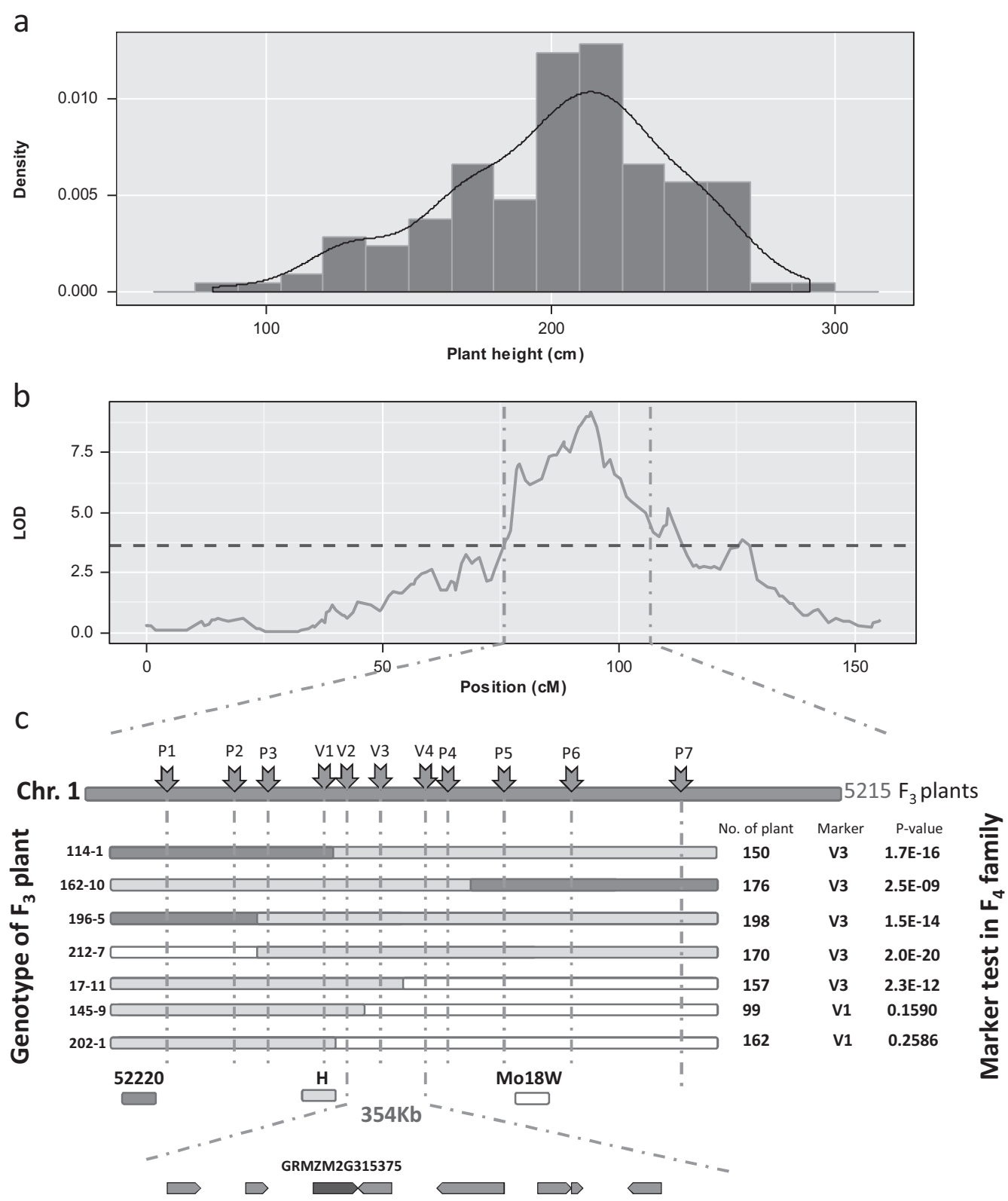

Fig. 3 Fine-mapping of major QTL of plant height on maize chromosome 1. a The density of plant height distribution in the $\mathrm{F}_{2}$ population. b QTL mapping identified a major QTL of plant height in the position from 80 to $110 \mathrm{cM}$ on maize chromosome 1; red dashed line signifies the significant threshold $(P<0.05)$ with 1000 permutations. c Fine-mapping of this major QTL of plant height in an $\mathrm{F}_{3: 4}$ population with 5215 individuals. Marker tests were performed in the $F_{3: 4}$

delimited in the segment between V2 and V4, which contained a 354-kb sequence on maize chromosome 1 (Fig. 3c).

\section{Comparative genomic analysis of $B r 2$ loci across different cereal species}

Annotation analysis revealed eight genes (Supplementary Table s1) located in this 354-kb fragment, which included the $B r 2$ gene encoding P-glycoprotein. Among these 8 population to test the correlation of plant height and molecular markers. Dark blue, light blue and white bars represent the genomic fragments of homozygous 52220 and heterozygous and homozygous Mo18W genotypes, respectively. The recombination break point was set in the middle of two flanking markers in each zygote. The arrows represent the molecular markers for fine mapping (Color figure online)

genes, only the expression of the $\mathrm{Br} 2$ gene was detected in the stalk based on RT-PCR (Supplementary Figure s1). We next conducted comparative genomic analysis among maize, sorghum, rice and foxtail millet for which the genomes had been completely sequenced. Comparative mapping identified a syntenic block containing four conserved fragments on sorghum chromosome 7 , rice chromosome 8 and foxtail millet chromosome 6 (Fig. 4c and Supplementary Table s2). In this syntenic block, despite several 
Fig. 4 Comparative genomic analysis of maize $B r 2$ locus. a Dot plot of highly conserved genes on maize chromosome 1 from 197.5 to $207.5 \mathrm{Mb}$ and sorghum chromosome 7 from 50 to $60 \mathrm{Mb}$; blue and red dots represent highly conserved and $B r 2$ genes, respectively. b QTL mapping revealed a major QTL of plant height corresponding to the sorghum $d w 3$ locus on sorghum chromosome 7; the $\mathrm{Br} 2$ gene was immediately under the QTL peak. Red dashed line represents the threshold $(P<$ $0.05)$. c Maize qpal contained a 241-bp deletion in the fifth exon, whereas sorghum $d w 3$ harbored an over 800-bp insertion in the similar position as that of maize qpal allele. Orange and blue bars signify exons; orange and blue lines indicate introns.

Triangles represent deletion and insertion. Two $\mathrm{ABC}$ transporter type I transmembrane (119-443 aa and 787-1107 aa) and two Ploop NTPase (455-701 aa and 1123-1359 aa) domains are shown on the bars. d Both $b r 2$ gene in maize (qpal) and sorghum $(d w 3)$ controlled the elongation of the internode of the stalk. The RIL plants with wild-type $\mathrm{Br} 2$ gene in maize and sorghum are shown as controls. Scale bar, $20 \mathrm{~cm}$. e Allele frequencies of maize qpal and sorghum $d w 3$ (Color figure online) a
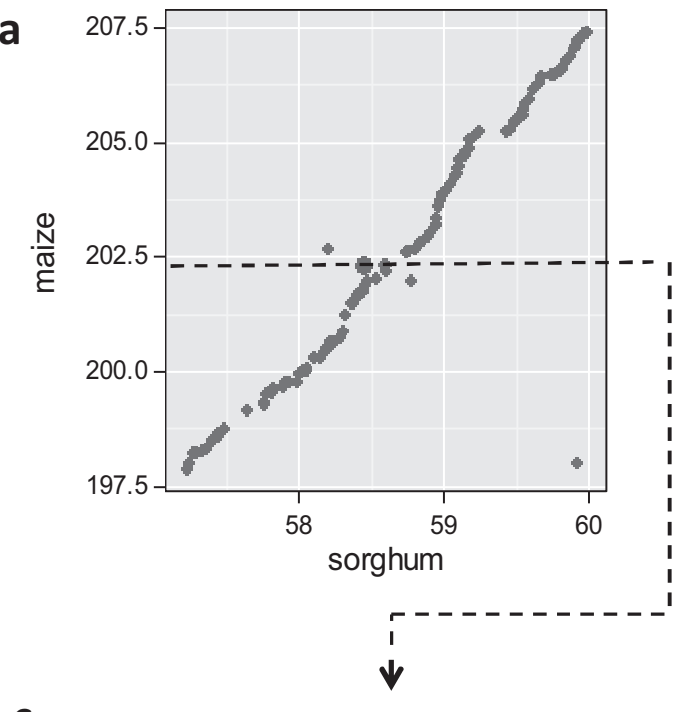

C

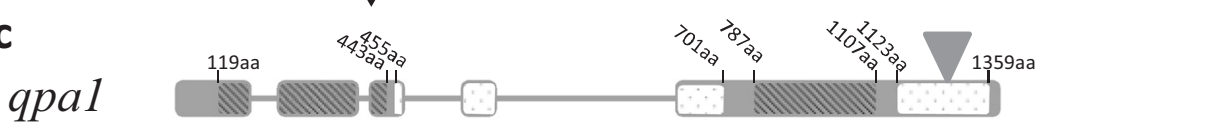

$d w 3$

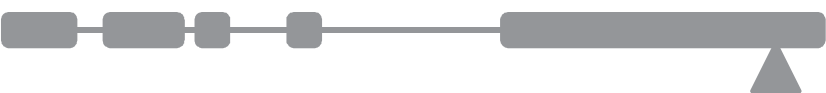

d
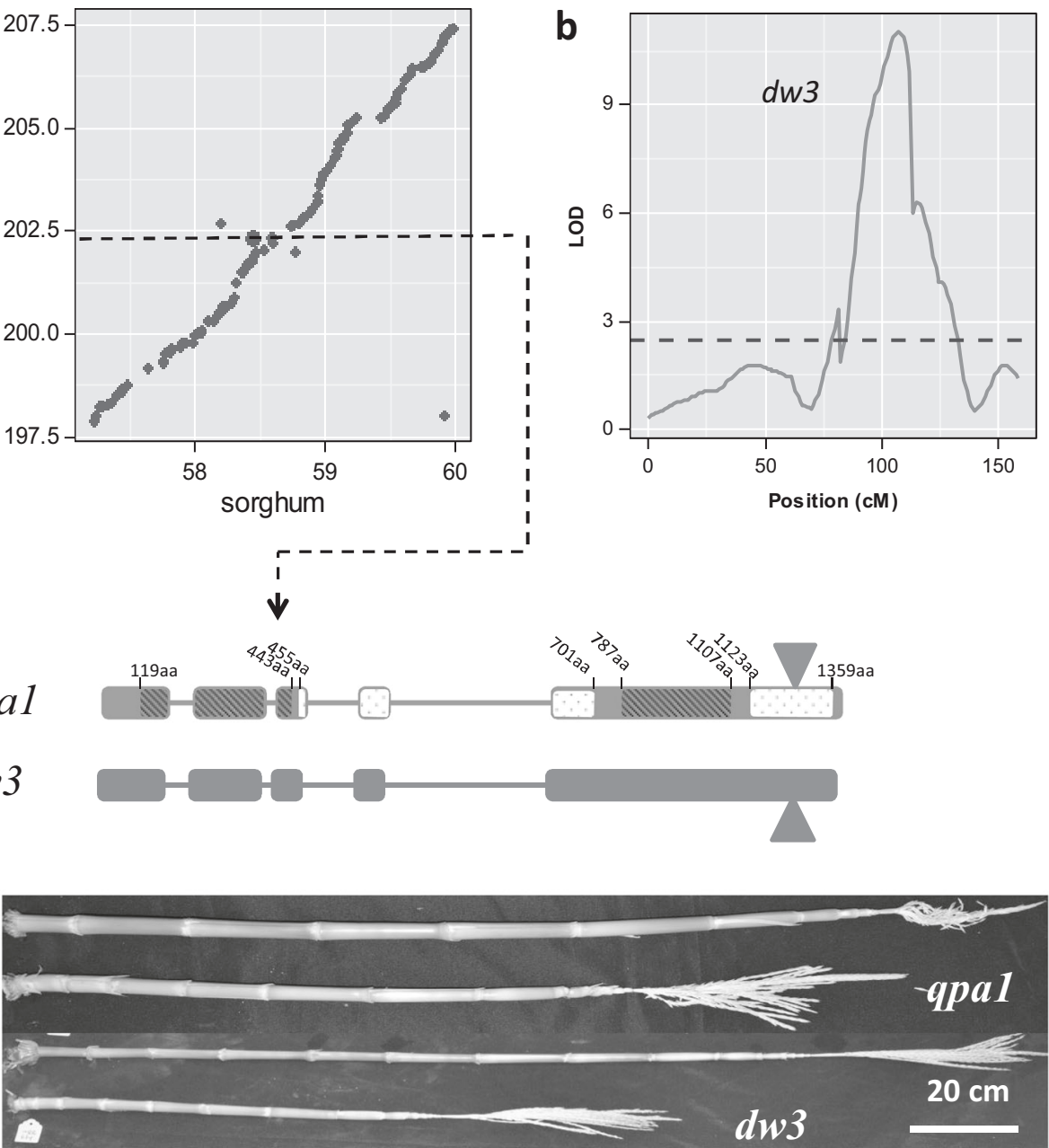

b

Position (cM)

$d w 3$

e

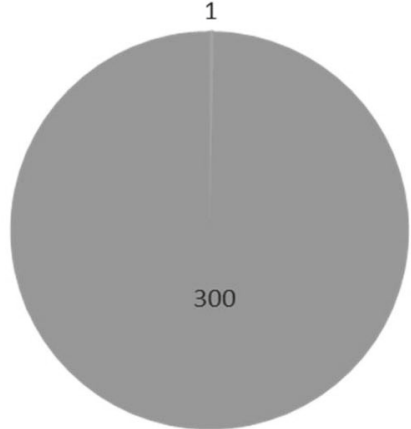

qpal

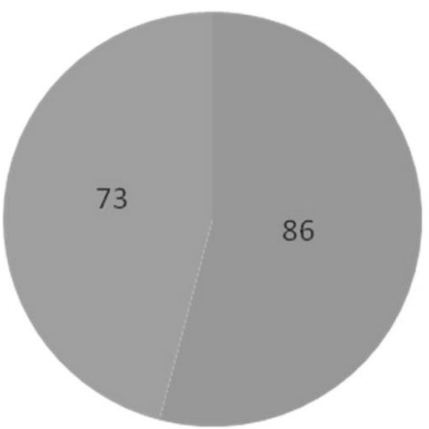

$d w 3$ translocations and reversions, the order of most genes in these fragments was in high collinearity among these four species. In sorghum, the major QTL for plant height accounted for $15 \%$ of the phenotypic variation and corresponded to the $d w 3$ locus in previous studies (Fig. 4b) (Brown et al. 2008)(Li et al. 2015). This QTL was identified in the syntenic fragment on chromosome 7 in a RIL population derived from the cross between a wild sorghum virgatum and a domesticated sorghum Tx623. Maize $\mathrm{Br} 2$ gene (GRMZM2G315375) corresponded to $d w 3$ (Sb07g023550) in sorghum, LOC_Os08g45030 in rice and Seita.6G253500 in foxtail millet. The rice $\mathrm{Br} 2$ orthologous gene was not located in the syntenic block because of an ancient translocation across different chromosomes. 
a
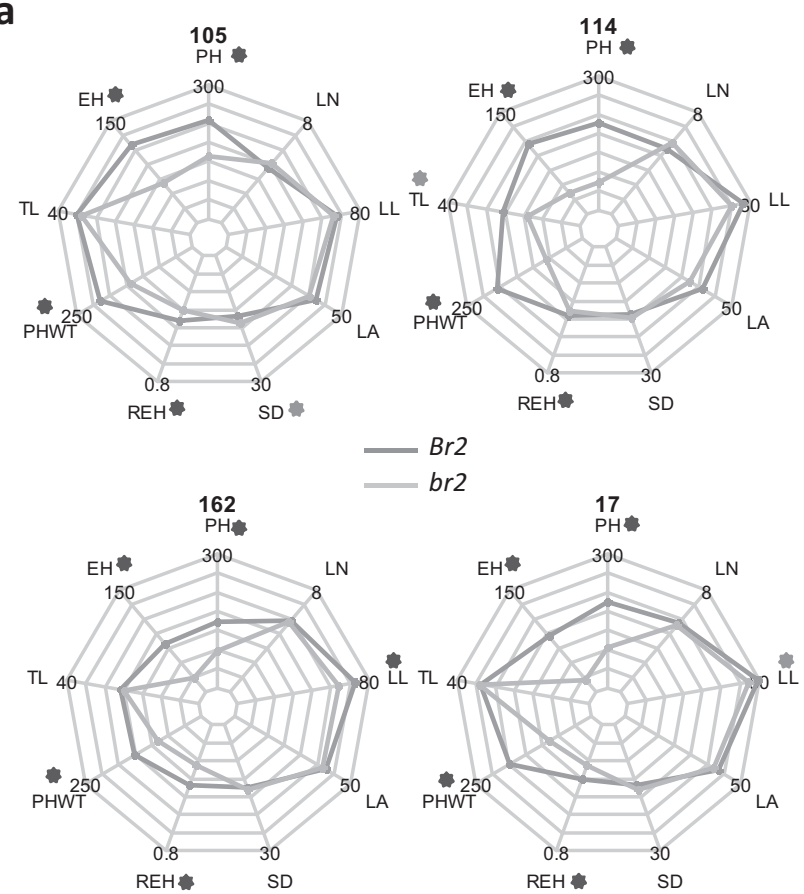

Fig. 5 Large phenotype comparisons between $\mathrm{Br} 2$ and $b r 2$ RILs with similar genetic background in maize. a Radar plots of plant architecture traits including plant height $(\mathrm{PH})$, ear height $(\mathrm{EH})$, plant height without tassel (PHWT), the relative ear height (REH, ear height/plant height), tassel length (TL), leaf number (LN), leaf length (LL), leaf angle (LA) and stalk diameter (SD) from $4 \mathrm{~F}_{4}$ families $(105,114,162$ and 17). b Radar plots of ear traits including husk leaf number (HLN),

\section{A new rare allele of $B r 2$ in maize}

To confirm whether $B r 2$ was responsible for this major QTL of plant height in maize in this study, sequence analysis of the $B r 2$ gene was then performed. The $B r 2$ gene contained a 6655-bp fragment with five exons and four introns in the tall parent line Mo18W, and the 4146-bp coding sequence (CDS) encoded a protein with 1381 amino acid residues (aa) (Fig. 4c), which contained two ABC transporter type I transmembrane (119-443 aa and 787-1107 aa) and two Ploop NTPase (455-701 aa and 1123-1359 aa) domains determined using the Interpro domain scan (http://www.ebi. ac.uk/interpro). By contrast, the $b r 2$ gene in the short parental line 52220 contained a 241-bp deletion in the fifth exon from 6376 to $6617 \mathrm{bp}$, which led to the loss of part of the second P-loop NTPase domain in translation (Fig. 4c). Interestingly, an over 800-bp insertion at the similar position of the fifth exon to which the 241-bp deletion was present in maize $b r 2$ gene also disturbed the second P-loop NTPase domain in the sorghum $b r 2$ gene, corresponding to the $d w 3$ locus from the short parent line Tx623 (Fig. 4c). Further investigation of plant height from the RILs with wild and mutant-type $\mathrm{Br} 2$ genes under similar genetic background revealed that the $B r 2$ gene controlled the b
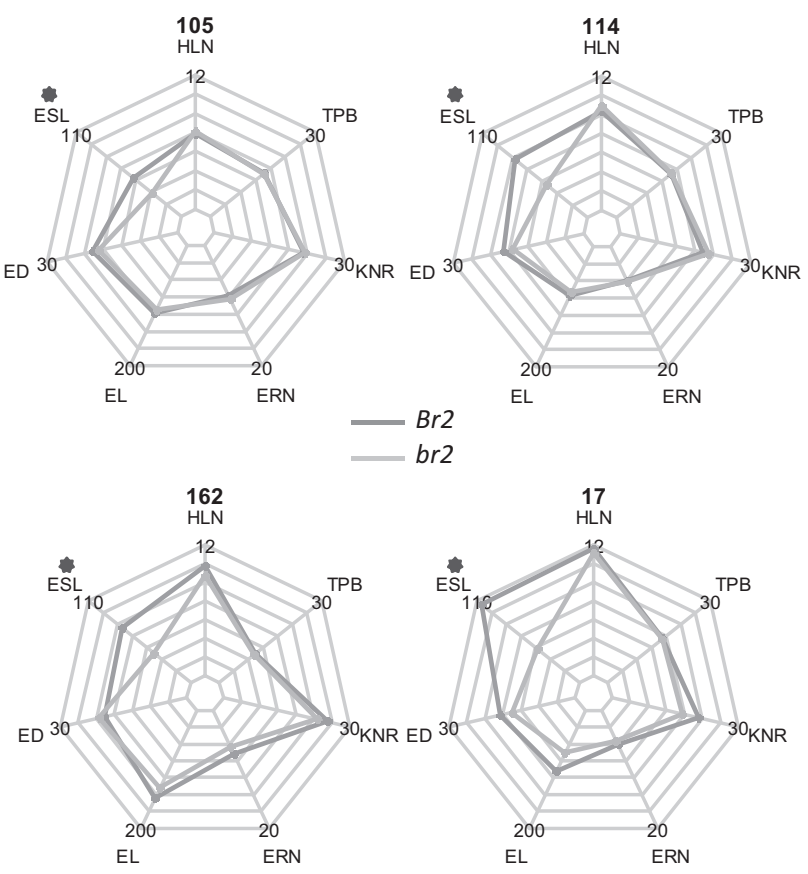

tassel primary branch number (TPB), ear shank length (ESL), ear row number (ERN), ear length (EL), ear diameter (ED) and kernel number per row (KNR) from the four families. Student's $t$-test was performed for each trait between the $B r 2$ and $b r 2$ lines $(n>25)$; red and orange stars indicate highly significant $(P<0.01)$ and significant $(P<0.05)$ signals, respectively. The blue numbers on the spider web represent the maximum values of traits (Color figure online)

elongation of the internode of stalks in maize and sorghum, which is consistent with previous studies (Fig. 4e). These results suggested that the $B r 2$ gene with the 241-bp deletion caused the reduction of plant height in the parental line 52220. In sorghum, the insertion in the $d w 3$ locus was present in 73 of 159 global sorghum accessions, suggesting that this insertion allele had been extensively applied to reduce plant height in sorghum (Fig. $4 \mathrm{~d}$ and Supplementary Table s3). However, the 241-bp deletion was only detected in 52220 in the scan of 311 maize accessions including 76 landraces and 235 inbred lines, indicating that the deletion had not yet been used in maize breeding (Fig. 4d and Supplementary Table s4).

\section{Large phenotype comparisons for new allele of br2 (qpa1)}

To estimate the potential for application of maize $b r 2$ gene with the 241-bp deletion in maize breeding, we performed large phenotypic measurements for four sets of RILs with similar genetic background from the $\mathrm{F}_{4}$ population (see Materials and Methods); each of which contained wild-type $B r 2$ and mutant-type $b r 2$ with the 241-bp deletion (Fig. 5). Plant height, ear height, plant height without tassel and the 

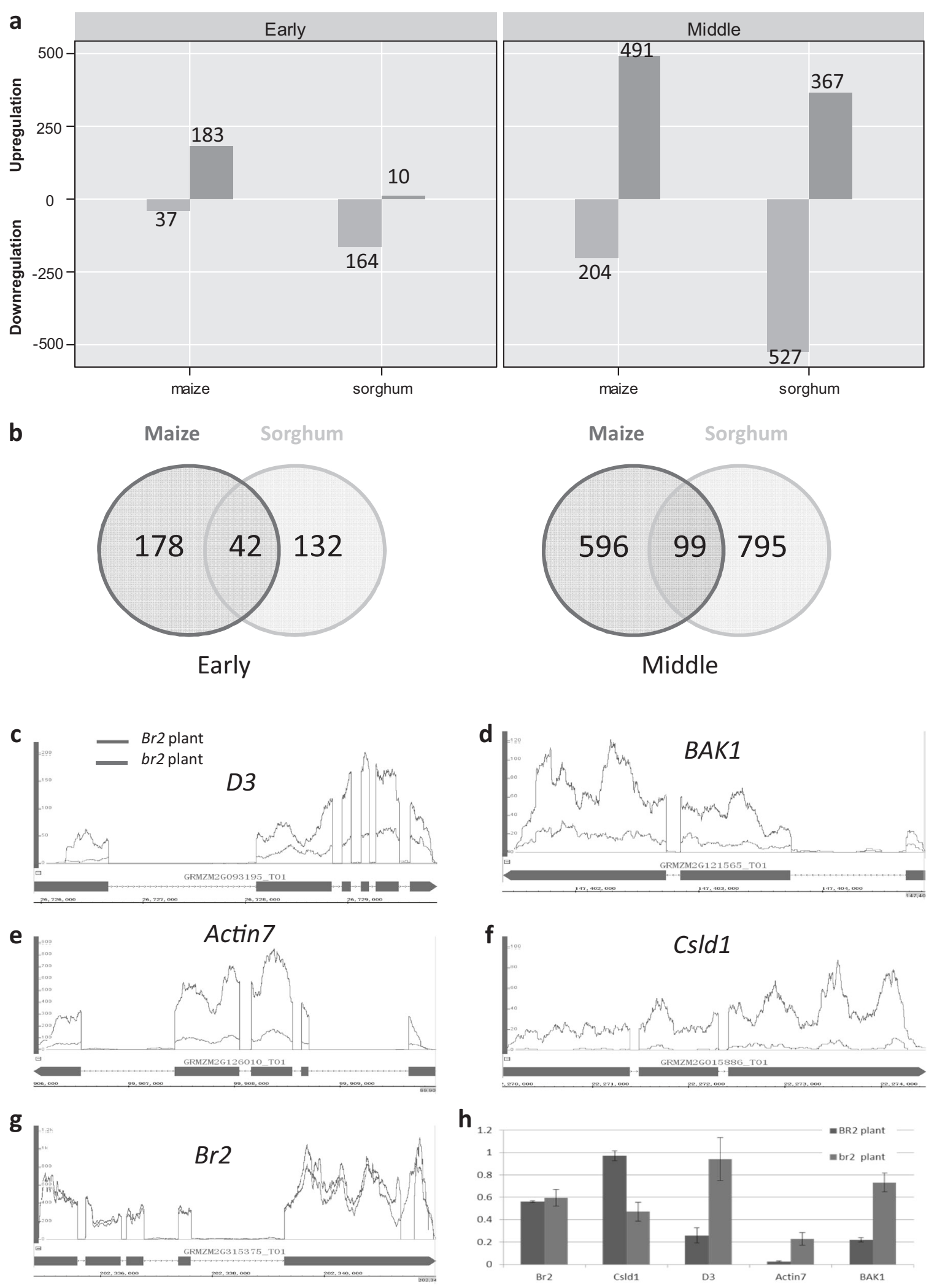

relative ear height (ear height/plant height) decreased significantly $(P<0.01)$ in the $b r 2$ lines compared with the $\mathrm{Br} 2$ lines in these four families. The reduced plant height and ear

height reached 90 and $50 \mathrm{~cm}$, respectively, in family 114 (Fig. 5a). However, leaf number above ear and tassel length were generally not significantly different between the $\mathrm{Br} 2$ 
Fig. 6 Comparative RNA-seq analysis. a The number of misregulation genes in the early and middle jointing stages. The numbers of upregulated and down-regulated gene are shown on the bar chart. b Differentially expressed gene number in the early and middle jointing stages. c-g The read coverage of five genes shown from maize RNAseq data. The transcripts of $D 3, B A K 1$ and Actin 7 were negatively regulated, whereas that of $C s d l l$ was positively regulated. The y-axis represents the read coverage, and the $x$-axis signifies the gene structure. Red line, $B r 2$ plant; blue line, $b r 2$ plant. h The transcript patterns of five genes were confirmed through real-time RT-PCR. Student's $t$ tests showed that not significant and significant transcription differences were present in $B r 2(P=0.61)$ and $C s l d 1, D 3, A c t i n 7$ and BAK1 genes $(P<0.05)($ Color figure online)

and $b r 2$ lines from all four families, except for $114(P=$ 0.03 for tassel length). Leaf length and leaf angle showed reduced values in the $b r 2$ lines from all four families, and a highly significant difference of leaf length was only observed in family 162. The trait of stalk diameter increased in the $b r 2$ lines from all four families, although a significant increase in stalk diameter $(P=0.03)$ was only detected in the $b r 2$ lines from family 105 (Fig. 5a). Further comparisons of the 10 ear traits revealed that, with the exception of ear shank length $(P<0.01)$, the traits of tassel primary branch number, husk leaf number, ear length, ear row number, kernel number per row, and ear diameter were not significantly reduced in the $b r 2$ lines compared with the $B r 2$ lines from all four families (Fig. 5b and Supplementary Figure s2). Lodging in maize is determined by four factors: plant height, ear height, stalk diameter and stalk stiffness. Therefore, the reduced plant height and ear height and slightly increased stalk diameter and no clearly reduced traits of ear in the $b r 2$ lines. These results signified that the br2 gene with 241-bp deletion could efficiently reshape the plant architecture and may provide strong resistance to lodging in maize. Therefore, this major QTL of plant architecture on chromosome 1 was then designated as qpal

\section{Comparative RNA-seq analysis of the $B r 2$ and br2 RILs in maize and sorghum}

To determine whether the $B r 2$ gene controls plant height, comparative RNA-seq analysis between maize and sorghum was then conducted from the stalks of both RIL plants with wild-type $B r 2$ and mutant-type $b r 2$ genes in the early and middle jointing stages (Fig. 6, see Materials and Methods). In the early jointing stage, the number of the DE genes was 220 and 174 , which accounted for approximately $1 \%$ of the total number of expressed genes of maize and sorghum, respectively (Fig. 6a). Among these DE genes, 183 genes were up-regulated and 37 genes were down-regulated in maize, whereas 10 genes were up-regulated and 164 genes were down-regulated in sorghum (Fig. 6a). In the middle stage, the number of DE genes increased tremendously in both maize and sorghum, with the number of DE genes reaching 695 and 894, respectively. Of these DE genes, 491 genes were up-regulated and 204 genes were downregulated in maize, whereas 367 were up-regulated and 567 were down-regulated in sorghum (Fig. 6a). More of the DE genes were up-regulated than down-regulated in maize in both early and middle jointing stages; however, the reverse pattern was observed in sorghum, with more downregulated than up-regulated genes (Fig. 6a). The number of differentially co-expressed genes between maize and sorghum was 42 in the early stage, which increased to 99 in the middle stage of jointing (Fig. 6b). Additionally, based on real-time PCR, the expression of $B r 2$ in the stalk was low in the early stage and then clearly increased in the middle stage of jointing (Supplementary Figure s3). These results suggested that the $B r 2$ gene primarily functioned in the middle stage of jointing.

Among the 99 differentially co-expressed genes affected by the $b r 2$ gene between maize and sorghum in the middle stage of jointing (Supplementary Table s5), four plant height genes D3 (GRMZM2G093195), Csld1 (GRMZM2G015886), Actin7 (GRMZM2G126010) and $B A K 1$ (GRMZM2G121565) coding cytochrome P450 88A1, cellulose synthase-like family, IAA transporting and BR synthesis proteins, respectively, were used for further study. To explore the interaction of the $B r 2$ gene with the four plant height genes, D3, Csld1, Actin 7 and $B A K 1$, we compared the transcription of these five genes from the data of comparative RNA-seq. Br2 encodes P-glycoprotein, which controls the polar transport of auxin; however, whether the Br2 gene can affect GA and BR biosynthesis and other auxin regulators remains poorly understood. The GA biosynthesis gene D3 exhibited a similar level of expression $(P>0.4)$ in the plants with $B r 2$ and $b r 2$ in the early stage of jointing in both species; however, then the transcription significantly increased in maize $(P=0.04)$ and sorghum $b r 2(P=0.005)$ plants in the middle stage of jointing, whereas no significant difference in expression between the plants with $B r 2$ and $b r 2$ was detected in maize and sorghum in both stages (Fig. 6 and Supplementary Figure s4). For $B A K 1$ and Actin7, similarly to D3, the expression was equal between the plants with $B r 2$ and $b r 2$ in the early stage in both species, and the transcriptions were then up-regulated in the middle stage in the $b r 2$ samples of both species (Supplementary Figure s4). These results indicated that the $B r 2$ gene might negatively regulate the biosynthesis of GA and BR and also the auxin efflux regulator. By contrast, the Csldl gene showed lower expression in the plants with $b r 2$ than those with $B r 2$ in the middle stage in both species, indicating that the $B r 2$ gene may positively control cellulose synthesis (Fig. 6 and Supplementary Figure s4). The transcript patterns of these 
five genes were consistent with those from real-time RTPCR in maize (Fig. 6h). All the above results suggested that $B r 2$ interacted with $D 3, B A K 1$, Actin 7 and Csld1 to regulate plant height.

\section{Discussion}

\section{The qpa1 can efficiently reshape plant architecture in maize breeding}

Plant height plays a central role in the reshaping of plant architecture. In rice and wheat, the semi-dwarf genes $s d l$ and $R h t l$ were widely and rapidly applied in the first green revolution. The rapid application of both genes was based on two advantages: both semi-dwarf genes greatly reduced plant height with only a single locus, and both genes had little influence on yield traits such as panicle length, panicle branch number and grain size. However, the reshaping of maize plant height to a moderate plant stature requires a long time, because breeders generally must combine many QTLs of plant height with small genetic effects. The semidwarf allele with large genetic effect and few negative yield traits has not been reported in maize. In the B73 $\times$ Ki11 RIL population, we identified a QTL corresponding to the $B r 2$ locus with minor genetic effect (Supplementary Figure s5). Several other alleles of maize $b r 2$ have been also identified, which include three $\mathrm{Mu}$ insertions in the exon and intron (Multani et al. 2003), one SNP variant in the exon (Xing et al. 2015) and one 8-bp deletion (Pilu et al. 2007) in the exon. The SNP variant and 8-bp deletion (Pilu et al. 2007) greatly reduce plant height, ear height and mildly decrease ear length, and these two alleles have good potential to reshape plant architecture without the loss of yield in the hybrid. In this study, we identified a new allele of $b r 2$ (qpal), which contributed large and small genetic effects on plant height and yield traits, respectively. Sorghum orthologous $d w 3$ gene corresponding to our qpal in maize has been widely applied in sorghum breeding, suggesting that our qpal also has good potential to modify plant architecture without yield loss, including effects on plant height, stalk diameter, leaf length and leaf angle, which enable high-density planting in maize.

\section{The modified fine-mapping method can greatly accelerate the cloning of major QTL in cereal}

In previous studies, a fine mapping of a QTL in a crop generally begins with the construction of an advanced backcrossed population, which is then followed by the construction of a large population in which the QTL has been transferred into a Mendelian factor. This fine-mapping strategy requires a long time (often more than 5 years)
(Bernacchi et al. 1998). Association mapping can generally delimit the common allele into a very short genomic fragment. However, association mapping has little power to detect a rare allele. In this study, qpal was a new rare allele of the $B r 2$ gene from 311 global maize accessions. The QTL mapping of plant height was performed in the $F_{2: 3}$ population with a high-density map of SNPs based on GBS analysis, and fine-mapping was subsequently conducted in the $\mathrm{F}_{3: 4}$ population with a marker test in the $\mathrm{F}_{4}$ families. Our modified strategy of progeny test in the fine-mapping of QTL has successfully identified qpal in maize and another major QTL of flowering time ( $H d l$ ) in sorghum (Liu et al. 2015). Thus, a QTL study from the initial QTL mapping to final fine mapping can be completed within 2 years using our strategy. Therefore, our strategy can be applied to rapidly clone such rare or major QTL as qpal in a crop.

\section{The qpa1 and 99 differentially co-expressed genes from comparative RNA-seq provide good candidates for further study and breeding}

Our comparative genomic analysis revealed that qpal corresponded to $d w 3$ in sorghum, LOC_Os08g45030 in rice, and Seita.6G253500 in foxtail millet. Rice $s d l$ and wheat Rhtl are generally used to reduce plant height in most breeding programs; however, these wide applications will undoubtedly narrow the genetic diversity. In this study, we identified the qpal with a deletion at the fifth exon from 6376 to $6617 \mathrm{bp}$, and an insertion was also at the similar position in sorghum $d w 3$. No significant transcript difference was detected in the plants with $B r 2$ and qpal. Unlike other alleles of $B r 2$ with large negative effects on yield, both qpal and $d w 3$ resulted in the loss of part of the second P-loop NTPase domain in translation and may lead to the loss of part of gene function in causing the semi-dwarf phenotype. Thus, both qpal and $d w 3$ alleles provide a good position target for genomic editing through CRISPR (Cong et al. 2013) to create new semi-dwarf plant material for rice, wheat and foxtail millet. Comparative RNA-seq analysis between maize and sorghum efficiently controlled the transcript noise and 99 differentially co-expressed genes were identified, which included 4 genes under natural selection (Supplementary Table s5) and 4 plant height genes (D3, BAKl, Actin7 and Csld1). These gene sources can be edited using CRISPR to create new plant materials for further study of the genetic basis of plant height and for breeding.

Acknowledgements This work was supported by National Natural Science Foundation of China (91735305 and 91335108 to Z.L.), the National Key Research and Development Program of China (2016YFD0101803 to Z.L.) and Chinese Thousand Youth Talents Program. 
Author contributions ZL designed research. WL, XZ, ZZ, and HL conducted the research. WL, XZ, ZZ, HL, and ZL analyzed the data. LW and ZL wrote the manuscript.

\section{Compliance with ethical standards}

Conflict of interest The authors declare that they have no conflict of interest.

\section{References}

Bensen RJ, Johal GS, Crane VC, Tossberg JT, Schnable PS, Meeley $\mathrm{RB}$ et al. (1995) Cloning and characterization of the maize An1 gene. Plant Cell 7(1):75-84

Bernacchi D, Beck-Bunn T, Eshed Y, Lopez J, Petiard V, Uhlig J et al. (1998) Advanced backcross QTL analysis in tomato. I. Identification of QTLs for traits of agronomic importance from Lycopersicon hirsutum. Theor Appl Genet 97(3):381-397

Bolger AM, Lohse M, Usadel B (2014) Trimmomatic: a flexible trimmer for Illumina sequence data. Bioinformatics 30 (15):2114-2120

Broman KW, Wu H, Sen S, Churchill GA (2003) R/qtl: QTL mapping in experimental crosses. Bioinformatics 19(7):889-890

Brown PJ, Rooney WL, Franks C, Kresovich S (2008) Efficient mapping of plant height quantitative trait loci in a sorghum association population with introgressed dwarfing genes. Genetics 180(1):629-637

Cong L, Ran FA, Cox D, Lin SL, Barretto R, Habib N et al. (2013) Multiplex genome engineering using CRISPR/Cas systems. Science 339(6121):819-823

Dobin A, Davis CA, Schlesinger F, Drenkow J, Zaleski C, Jha S et al. (2013) STAR: ultrafast universal RNA-seq aligner. Bioinformatics 29(1):15-21

Duvick DN (2005) Genetic progress in yield of United States Maize (Zea Mays L.). Maydica 50(3-4):193-202

Elshire RJ, Glaubitz JC, Sun Q, Poland JA, Kawamoto K, Buckler ES, Mitchell SE (2011) A robust, simple genotyping-by-sequencing (GBS) approach for high diversity species PLoS One 6(5):e19379

Freeling M (2009) Bias in plant gene content following different sorts of duplication: tandem, whole-genome, segmental, or by transposition. Annu Rev Plant Biol 60:433-453

Hedden P (2003) The genes of the Green Revolution. Trends Genet 19 (1):5-9

Huang XH, Feng Q, Qian Q, Zhao Q, Wang L, Wang AH et al. (2009) High-throughput genotyping by whole-genome resequencing. Genome Res 19(6):1068-1076

Kersey PJ, Allen JE, Armean I, Boddu S, Bolt BJ, Carvalho-Silva D et al. (2016) Ensembl Genomes 2016: more genomes, more complexity. Nucleic Acids Res 44(D1):D574-D580

Kinsella RJ, Kahari A, Haider S, Zamora J, Proctor G, Spudich G et al. (2011). Ensembl BioMarts: a hub for data retrieval across taxonomic space. Database Article ID bar 030: 1-9

Langmead B, Trapnell C, Pop M, Salzberg SL (2009) Ultrafast and memory-efficient alignment of short DNA sequences to the human genome Genome Biol 10(3):1-10

Li H, Durbin R (2009) Fast and accurate short read alignment with Burrows-Wheeler transform. Bioinformatics 25(14):1754-1760
Li X, Li XR, Fridman E, Tesso TT, Yu JM (2015) Dissecting repulsion linkage in the dwarfing gene Dw3 region for sorghum plant height provides insights into heterosis. Proc Natl Acad Sci USA 112(38): $11823-11828$

Liu H, Liu H, Zhou L, Zhang Z, Zhang X, Wang M et al. (2015) Parallel domestication of the heading date 1 gene in cereals. Mol Biol Evol 32(10):2726-2737

Livak KJ, Schmittgen TD (2001) Analysis of relative gene expression data using real-time quantitative PCR and the 2(T)(-Delta Delta C) method. Methods 25(4):402-408

McKenna A, Hanna M, Banks E, Sivachenko A, Cibulskis K, Kernytsky A et al. (2010) The Genome Analysis Toolkit: a MapReduce framework for analyzing next-generation DNA sequencing data. Genome Res 20(9):1297-1303

Multani DS, Briggs SP, Chamberlin MA, Blakeslee JJ, Murphy AS, Johal GS (2003) Loss of an MDR transporter in compact stalks of maize br2 and sorghum dw3 mutants. Science 302(5642):81-84

Nicol JW, Helt GA, Blanchard SG, Raja A, Loraine AE (2009) The Integrated Genome Browser: free software for distribution and exploration of genome-scale datasets. Bioinformatics 25 (20):2730-2731

Peiffer JA, Romay MC, Gore MA, Flint-Garcia SA, Zhang Z, Millard MJ et al. (2014) The genetic architecture of maize height. Genetics 196(4):1337-1356

Peng JR, Richards DE, Hartley NM, Murphy GP, Devos KM, Flintham JE et al. (1999) 'Green revolution' genes encode mutant gibberellin response modulators. Nature 400(6741):256-261

Pilu R, Cassani E, Villa D, Curiale S, Panzeri D, Cerino Badone F et al. (2007) Isolation and characterization of a new mutant allele of brachytic 2 maize gene. Mol Breed 20(2):83-91

Salazar AM (1985). Effects of mass selection for ear length in maize. Retrosp Theses Dissertations. 8745. http://lib.dr.iastate.edu/rtd/ 8745.

Sasaki A, Ashikari M, Ueguchi-Tanaka M, Itoh H, Nishimura A, Swapan D et al. (2002) Green revolution: a mutant gibberellinsynthesis gene in rice-new insight into the rice variant that helped to avert famine over thirty years ago. Nature 416 (6882):701-702

Trapnell C, Hendrickson DG, Sauvageau M, Goff L, Rinn JL, Pachter L (2013) Differential analysis of gene regulation at transcript resolution with RNA-seq. Nat Biotechnol 31(1):46-53

Winkler RG, Helentjaris T (1995) The maize Dwarf3 gene encodes a cytochrome P450-mediated early step in gibberellin biosynthesis. Plant Cell 7(8):1307-1317

Xing AQ, Gao YF, Ye LF, Zhang WP, Cai LC, Ching A et al. (2015) A rare SNP mutation in Brachytic2 moderately reduces plant height and increases yield potential in maize. J Exp Bot 66 (13):3791-3802

Yang N, Lu YL, Yang XH, Huang J, Zhou Y, Ali F et al. (2014). Genome wide association studies using a new nonparametric model reveal the genetic architecture of 17 agronomic traits in an enlarged maize association panel. PLoS Genet 10(9)

Yang XH, Gao SB, Xu ST, Zhang ZX, Prasanna BM, Li L et al. (2011) Characterization of a global germplasm collection and its potential utilization for analysis of complex quantitative traits in maize. Mol Breed 28(4):511-526 